\title{
IMPROVING PRODUCTIVE AND PHYSIOLOGICAL PARAMETERS OF BROILERS USING SOME LIGHT REGIMES AND FEED ADDITIVES
}

\author{
M.M. Soliman ${ }^{1}$, B.A. El-Neney ${ }^{1}$, M.A. Kalamah ${ }^{2}$ and A.K. Alm El-dein ${ }^{1}$ \\ 1- Animal Production Research Institute, Dokky, Giza, Egypt, 2- Department of \\ Poultry Production, Faculty of Agriculture, University of Menuofiya, Egypt,
}

\section{SUMMARY}

A total number of 360 one-day-old unsexed broiler chicks (Arbor Acres) was used in a 42-day experiment to investigate the effect of lighting regimes and Bio-Tonic level in diet on chick performance. Chicks were randomly divided into three equal groups according to light regimes: (L1) continuous (23L:1D), (L2) constant (15L:9D) and (L3) intermittent (8L:4D:8L:4D). Each light program was divided into four groups according to level of Bio-Tonic in diet as follow, (B1) 0.0, (B2) 0.5, (B3) 1.0 and (B4) $1.5 \mathrm{~kg} /$ ton diet. Chicks exposed to intermittent lighting had significantly higher $(P<0.05$ or $P<0.01)$ live body weight at 7 weeks of age and body gain during 4-7 and 1-7 week periods than those exposed to continuous or constant lighting program. Chicks fed on B4 had significantly $(P<0.01)$ higher body weight at 7 wks than those fed on other diets at all ages studied. The differences among Bio-Tonic levels in body weight at 7 weeks and body gain during 1-7 week period were highly significant $(P<0.01)$. Feed consumption and feed conversion ratio of continuous lighting were significantly $(P<0.05$ or $P<0.01)$ greater than those of either constant or intermittent lighting treatments at all ages studied. The birds fed on B1 had significantly higher $(P<0.05)$ feed consumption and feed conversion ratio than those fed on other Bio-Tonic levels in diet during all periods studied. Chicks fed on B4 gave non-significantly greater percentages of dressing, liver, heart, gizzard and giblets than that fed on the other Bio-Tonic treatments. An opposite trend was true for abdominal fat $\%$ with B1 treatment. Birds exposed to constant lighting had ether extract \% significantly $(P<0.05)$ greater than those exposed to continuous and intermittent lighting programs. Chicks reared under intermittent gave significantly greater $(P<0.05$ or $P<0.01)$ liver protein \% and glycogen $(\mathrm{mg} / \mathrm{g})$ than that reared under both continuous and constant lighting programs. Chicks fed on B4 level had non-significantly lower liver lipids \% than other levels, and had greater liver protein $\%$ and liver glycogen $(\mathrm{mg} / \mathrm{g})$ than the other Bio-Tonic levels. Plasma total lipids were insignificantly higher of constant than those continuous or intermittent lighting programs. The chicks fed on B4 level had plasma total lipids and cholesterol lower, and plasma total protein, albumin, alkaline phosphatase activity, GOT, T3, T4 and $G H$ higher than that fed on the other levels of Bio-Tonic. There were significant differences $(P<0.05$ or $P<0.01)$ due to interaction effect between light regimes and Bio-Tonic level in the diet on body weight at 4 and 7 wk; body gain during 4-7 and 17 wh periods; feed consumption during all periods studied; feed conversion ratio during 1-4 and 4-7 wk periods; E.E.\%; liver protein \%; percentages of dressing and abdominal fat and plasma total protein, albumin, cholesterol, T3 and GH.

Issued by The Egyptian Society of Animal Production 
Keywords: Broilers, feed additives, light regimes, body weight, physiological

\section{INTRODUCTION}

Understanding the role of photoperiod regimens in production performance becomes increasingly important especially concerning the cost reduction of the management of broiler chicks. There have been attempts to investigate the advantages of using different light regimens rather that which is commonly used (23 hours of light and 1 hour of darkness daily). Classen and Riddell (1989) and Classen et al. (1991) concluded that the use of continuous or near continuous light should not be recommended for broiler chickens. Intermittent light had significantly greater body weights and better feed efficiency than that of continuous illumination (Weaver et al., 1982) and may be less stressful to the birds than continuous light (Buckland et al., 1971).

The manipulation of photoperiods in raising broiler chicks has been largely done to maximize body weight and improve feed efficiency (Classen and Riddell, 1989). Long photoperiods or constant lighting are believed to increase feed consumption due to continuous access to feed. Consumption of feed is almost entirely restricted to the period of light, with a peak in feed consumption at the beginning or at the end of the photoperiod (Savory, 1980). Buyse et al. (1993) showed that the amount of feed consumed during the dark period is less than $1 \%$ of that during the light period. Therefore, feed consumption varies according to lighting schedule.

Recent experiments have shown that intermittent lighting regimens can reduce feed intake and improve feed efficiency (Buyse et al., 1996).

The inclusion of minor quantities of specific components of natural or synthetic origin into compound feed is a common practice in the animal and poultry feed industry. These components, which are called feed additives, can be classified into two categories: First, those additives which are essential for the biological function of the animal such as vitamins and trace elements. The second category includes additives which are not essential for biological function, but have demonstrated a positive effect upon the animal, including growth promoters, metabolic modifiers and probiotics (Namur et al., 1988).

Therefore, this experiment aimed to study the effect of different lighting regimes with using certain levels of herbal extraction (Bio-Tonic) on the growth, blood and carcass traits of broiler chicks.

\section{MATERIALS AND METHODS}

A total number of 360 one-day-old commercial Arbor Acres broiler chicks was used in these experiments. Chicks were brooded in floor pens. They were fed during the first four weeks of age on a basal starter ration containing $23 \%$ crude protein and $3021 \mathrm{Kcal} \mathrm{ME} / \mathrm{kg}$ and from 5 to 7 weeks of age on a finisher diet containing $21 \%$ crude protein and $2995 \mathrm{Kcal} \mathrm{ME} / \mathrm{kg}$ (Table 1).

Chicks were wing-banded and weighed individually, then randomly divided into three equal groups according to light regimes, the first exposed to continuous light had 23 hours of light and one hour darkness (23 L: $1 \mathrm{D})$, the second exposed to constant light had 15 hours of light and 9 hours of darkness (15 L: 9 D) and the third exposed to intermittent light had 8 hours light then 4 hours darkbess then 8 hours 
light then 4 hours darkness (8 L: 4 D: $8 \mathrm{~L}: 4 \mathrm{D}$ ). Each light program included four groups according to Bio-Tonic (a mixture of fermented and dried extracts of several herbs and edible plants in addition to dried condensed corn distillers, Abdel-Malak et al., 1995) in diet as follow, (B1) 0.0, (B2) 0.5 , (B3) 1.0 and (B4) $1.5 \mathrm{~kg} /$ ton diet.

Table 1. Composition and calculated analysis of the basal experimental diet

\begin{tabular}{lcc}
\hline Ingredients & Starter diet & Finisher diet \% \\
\hline Yellow corn & 57 & 65 \\
Soybean meal (44\%) & 23 & 12 \\
Corn gluten $(60 \%)$ & 10 & 11 \\
Wheat bran & 7 & 9 \\
Fat or oil & - & - \\
Limestone & 1 & 1 \\
Salt & 0.30 & 0.30 \\
L - Lysine & 0.20 & 0.20 \\
Di-Calcium Phosphate & 1 & 1 \\
$\quad$ DL - Methionine & 0.20 & 0.20 \\
Premix & 0.30 & 0.30 \\
Calculated analyses & & \\
Crude protein \% & 22.24 & 19 \\
ME, Kcal / Kg & 2974.2 & 3085.42 \\
C / P ratio & 133.7 & 162 \\
Ca \% & 0.88 & 0.92 \\
P \% & 0.43 & 0.46 \\
Lysine \% & 1.11 & 0.86 \\
Methionine \% & 0.61 & 0.58 \\
Cystine \% & 0.39 & 0.35 \\
\hline
\end{tabular}

"Premix: Vitamin and Mineral mixture supplied each kg diet:-

Vit A 1200 IU. Vit D3 2000 IU, Vit E 10mg, Vit K3 2mg, Vit B1 1mg, Vit B2 5mg, Vit B6 $1.5 \mathrm{mg}$, Vit B12 10mcg, Niacin $20 \mathrm{mg}$, Pantothenic acid $10 \mathrm{mg}$, Folic acid $1 \mathrm{mg}$, Choline chloride $250 \mathrm{mg}$, Biotin $50 \mathrm{mcg}$, Manganese $60 \mathrm{mg}$, Zinc $50 \mathrm{mg}$, Copper $5 \mathrm{mg}$, Iodine $0.3 \mathrm{mg}$, Iron $30 \mathrm{mg}$, Cobalt $0.1 \mathrm{mg}$ and Selenium $0.1 \mathrm{mg}$.

** According to NRC (1994).

Live body weight and feed consumption were biweekly recorded and body weight gain and feed conversion were then calculated. At the $7^{\text {th }}$ week of age, five birds from each treatment were randomly chosen and slaughtered. Eviscerated weight, giblets (liver, gizzard and heart), total edible parts (carcass and giblets) and abdominal fat were determined. Relative weights of the carcass traits to live body weight (LBW) were then calculated. Dressing percentage was computed as body weight (BW) of clean carcass plus edible viscera/LBW and as clean carcass/carcass weight. Liver samples were secured to determine liver lipids, protein and glycogen. Liver total lipids and total protein were determined using AOAC (1990), glycogen by Der Vies (1954) method.

At slaughtering, blood samples $(5 \mathrm{ml})$ were collected and centrifuged at $3000 \mathrm{rpm}$ for 20 minutes. Plasma produced was frozen at $-20^{\circ} \mathrm{C}$ till the time of chemical determination of plasma total protein, albumin, total lipids, cholesterol, activity of 
alkaline phophatase, Glutamic Oxaloacetic Transaminase (GOT), Glutamic Pyruvic Transaminase (GPT), Triiodothyronine $\left(\mathrm{T}_{3}\right)$, Thyroxine $\left(\mathrm{T}_{4}\right)$ and growth hormone (GH) using commercial kits.

Recorded data were subjected to statistical analysis using SPSS (1984). Data as percentages were transformed before analysis. The following model was used:

where :

$$
\mathrm{Y}_{\mathrm{ijk}}=\mu+\mathrm{L}_{\mathrm{i}}+\mathrm{B}_{\mathrm{j}}+(\mathrm{LxB})_{\mathrm{ij}}+\mathrm{e}_{\mathrm{ijk}} \text {. }
$$

$\mathrm{Y}_{\mathrm{ijk}}=$ Observation. $\quad \mu=$ General mean.

$\mathrm{L}_{\mathrm{i}}=$ Light regime effect. $\quad \mathrm{B}_{\mathrm{j}}=$ Bio-Tonic effect.

$(\mathrm{LxB})_{\mathrm{ij}}=$ The interaction effect between light regime and Bio-Tonic treatment.

$\mathrm{e}_{\mathrm{ijk}}=$ Random error.

\section{RESULTS AND DISCTION}

\section{Live body weight and weight gain}

Data presented in Table (2) show means of live body weight and weight gain of broiler chicks exposed to continuous, constant and intermittent lighting regimes from 1 up to 7 weeks of age. Chicks exposed to intermittent or continuous lighting had significantly $(\mathrm{P}<0.01)$ higher live body weights at 7 weeks of age than that exposed to constant lighting program. However, live body weights at 1 and 4 week of age were nearly similar. Body weight gain in chicks exposed to intermittent lighting was greater than that exposed to either continuous or constant lighting program, the differences in this respect, were significant $(\mathrm{P}<0.01)$ during 4-7 and significant $(\mathrm{P}<0.05)$ during 1-7 between intermittent and constant lighting programs (Table 2). This finding agree with the results of Al-Homidan (1994), Renden et al. (1996), Laster et al., (1999) and Othani and Lesson (2000). El- Neney (2003) showed that under intermittent light birds had significantly heavier body weight than those of continuous light and constant light. Intermittent light birds had the highest daily gain. The lowest one was the constant light regime.

On the other hand, Al-Homidan (1994), Buys et al. (1998) and Al-Homidan and Petchey (2001) observed non significant difference between light regimes on body weight at $7 \mathrm{wk}$ of age. Also, Kalamah (2002) found that broiler chicks reared under continuous lighting had significantly $(\mathrm{P}<0.01)$ higher body weights than those grown under either constant or intermittent lighting regime; also the weekly body weight gain was greater for birds under continuous lighting than those under constant or intermittent lighting regime. However, Smith (1994) found that, photo schedule (23L: $1 \mathrm{D}$ and $16 \mathrm{~L}: 8 \mathrm{D})$ had no effect on body weight gain.

Chicks fed on B4 $(1.5 \mathrm{~kg} /$ ton) Bio-Tonic plus diet had higher body weight than that fed on other diets at all ages studied. The differences among Bio-Tonic levels in body weight were highly significant $(\mathrm{P}<0.01)$ at 7 and not significant at 1 and 4 weeks of age (Table 2).

Body weight gain in chicks fed on B4 was greater than the other Bio-Tonic treatments, the differences in this respect, were highly significant $(\mathrm{P}<0.01)$ during 1-7 (Table 2). This finding agrees with the results of Abdel-Malak et al. (1995) who found that adding Bio-Tonic to broiler finisher diets as a growth promoter supplement improved live body weight and weight gain. Moreover, supplementing Bio-Tonic in broiler diets increased significantly live body weight and daily weight 
gain $(\mathrm{P}<0.01$ or 0.001$)$ at marketing age (Bardley et al., 1994; El-Gendi et al, 1994, Osman, 1996 and Tawfeek and Marai, 1997).

The effect of interaction between light regimes and Bio-Tonic level in diet on live body weight at 7 weeks of age and body weight gain during 1-7 weeks period was significant $(\mathrm{P}<0.01)$, significant $(\mathrm{P}<0.05)$ for live body weight at 4 weeks and body weight gain during 4-7 weeks period (Table 2). Moreover the highest value of both body weight (at 4 and 7 weeks) and body weight gain (during 4-7weeks and 1-7 weeks) was in treatment L3 x B4.

Table 2. Effect of light regimes and bio-tonic (B) level on body weight and body weight gain

\begin{tabular}{|c|c|c|c|c|c|c|}
\hline \multirow[t]{2}{*}{ Classification } & \multicolumn{3}{|c|}{ Body weight $(\mathrm{g})$} & \multicolumn{3}{|c|}{ Body weight gain $(\mathrm{g})$} \\
\hline & $1 \mathrm{WK}$ & $4 \mathrm{WK}$ & $7 \mathrm{WK}$ & $1-4 \mathrm{WK}$ & 4-7WK & $1-7 \mathrm{WK}$ \\
\hline \multicolumn{7}{|c|}{ Effect of light regimes } \\
\hline (L1) Continuous & 104 & 724 & $1880 \mathrm{a}$ & 620 & $1156 \mathrm{a}$ & $1776 \mathrm{a}$ \\
\hline (L2) Constant & 101 & 717 & $1739 b$ & 616 & $1022 b$ & $1638 b$ \\
\hline (L3) Intermittent & 106 & 779 & $1943 a$ & 673 & $1164 a$ & $1837 \mathrm{a}$ \\
\hline SEM & 2.1 & 14.2 & 35.5 & 17.5 & 25.0 & 37.5 \\
\hline Probability & N.S & N.S & 0.01 & N.S & 0.01 & 0.05 \\
\hline \multicolumn{7}{|c|}{ Effect of Bio-Tonic levels } \\
\hline B1 (control) & 101 & 706 & $1777 \mathrm{~b}$ & 605 & 1071 & $1676 b$ \\
\hline$(0.5 \mathrm{Kg} /$ ton $) \mathrm{B} 2$ & 101 & 718 & $1781 b$ & 617 & 1063 & $1680 \mathrm{~b}$ \\
\hline B3 $(1.0 \mathrm{Kg} /$ ton $)$ & 104 & 748 & $1887 \mathrm{a}$ & 644 & 1139 & $1783 \mathrm{a}$ \\
\hline B4 $(1.5 \mathrm{Kg} / \mathrm{ton})$ & 108 & 787 & $1970 \mathrm{a}$ & 679 & 1183 & $1862 \mathrm{a}$ \\
\hline SEM & 3.1 & 15.1 & 37.1 & 17.2 & 23.5 & 38.5 \\
\hline Probability & N.S & N.S & 0.01 & N.S & N.S & 0.01 \\
\hline \multicolumn{7}{|c|}{ Interaction effect between light regimes and Bio-Tonic levels } \\
\hline LI x B1 & 100 & $700 \mathrm{a}$ & $1830 \mathrm{bc}$ & 600 & $1130 \mathrm{c}$ & $1730 \mathrm{c}$ \\
\hline L1 x B2 & 102 & $695 a$ & $1835 \mathrm{c}$ & 593 & $1140 \mathrm{c}$ & $1733 \mathrm{c}$ \\
\hline L1 x B3 & 104 & $720 \mathrm{ab}$ & $1906 d$ & 616 & $1186 \mathrm{c}$ & $1802 \mathrm{c}$ \\
\hline L1 x B4 & 108 & $780 c$ & $1950 d$ & 672 & $1170 \mathrm{c}$ & $1842 \mathrm{~cd}$ \\
\hline L2 x B1 & 99 & $665 a$ & $1690 \mathrm{a}$ & 566 & $1025 \mathrm{a}$ & $1591 \mathrm{a}$ \\
\hline L2 x B2 & 100 & $700 \mathrm{a}$ & $1709 a$ & 600 & $1009 a$ & $1609 a$ \\
\hline L2 x B3 & 101 & $732 \mathrm{ab}$ & $1765 b$ & 631 & $1033 \mathrm{ab}$ & $1664 a b$ \\
\hline L2 x B4 & 103 & $770 \mathrm{bc}$ & $1790 \mathrm{~b}$ & 667 & $1020 \mathrm{a}$ & $1687 b$ \\
\hline L3 x B1 & 103 & $752 b$ & $1810 \mathrm{~b}$ & 649 & $1058 \mathrm{~b}$ & $1707 \mathrm{bc}$ \\
\hline $\mathrm{L} 3 \times \mathrm{B} 2$ & 102 & $760 \mathrm{~b}$ & $1800 \mathrm{~b}$ & 658 & $1040 b$ & $1698 b$ \\
\hline L3 x B3 & 106 & $793 c$ & 1990de & 687 & $1197 \mathrm{~cd}$ & $1884 d$ \\
\hline L3 x B4 & 113 & $810 \mathrm{~d}$ & $2170 \mathrm{e}$ & 697 & $1360 \mathrm{e}$ & $2057 \mathrm{e}$ \\
\hline SEM & 3.5 & 17.5 & 40.1 & 18.5 & 27.5 & 40.0 \\
\hline Probability & N.S & 0.05 & 0.01 & N.S & 0.05 & 0.01 \\
\hline
\end{tabular}

N.S. Not significant

a,b,c,d and e means within the same column not having similar superscripts are significantly different $(\mathrm{P}<0.05)$

\section{Feed consumption and feed conversion ratio}

Table (3) shows that feed consumption of continuous lighting was significantly $(\mathrm{P}<0.01)$ greater than that of either constant or intermittent lighting treatments at all 
ages studied. The feed conversion ratio was significantly $(\mathrm{P}<0.05)$ higher for continuous than intermittent lighting program and non significant higher than constant lighting program at all periods studied as shown in Table (3). Similar results were reported by Buys et al. (1998) and Al-Homidan and Patchey (2001). However, El-Neney (2003) showed that daily feed consumption of continuous and intermittent chickens had significantly higher means than that of constant chickens $(P \leq 0.05)$ at different periods studied. Each bird under intermittent light consumed less feed than that reared under continuous light regime. These results suggest that birds reared under intermittent light regime were more efficient than those reared under continuous light.

Table 3. Effect of light regimes and bio-tonic (B) level on feed consumption (g) and feed conversion ratio (feed/gain, FCR)

\begin{tabular}{|c|c|c|c|c|c|c|}
\hline \multirow[t]{2}{*}{ Classification } & \multicolumn{3}{|c|}{ feed consumption (g) } & \multicolumn{3}{|c|}{$\begin{array}{l}\text { feed conversion ratio } \\
\text { (feed /gain, FCR) }\end{array}$} \\
\hline & $1-4 W K$ & 4-7WK & $1-7 \mathrm{WK}$ & $1-4 W K$ & 4-7WK & $1-7 \mathrm{WK}$ \\
\hline \multicolumn{7}{|c|}{ Effect of light regimes } \\
\hline (L1) Continuous & $1733^{\mathrm{a}}$ & $2927^{\mathrm{a}}$ & $4660^{\mathrm{a}}$ & $2.80^{\mathrm{a}}$ & $2.23^{\mathrm{a}}$ & $2.62^{\mathrm{a}}$ \\
\hline (L2) Constant & $1412^{\mathrm{b}}$ & $2204^{\mathrm{b}}$ & $3616^{b}$ & $2.29^{\mathrm{a}}$ & $2.16^{\mathrm{a}}$ & $2.21^{\mathrm{a}}$ \\
\hline (L3) Intermittent & $1314^{\mathrm{b}}$ & $2287^{\mathrm{b}}$ & $3601^{b}$ & $1.95^{\mathrm{b}}$ & $1.96^{\mathrm{b}}$ & $1.96^{\mathrm{b}}$ \\
\hline SEM & 10.3 & 12.5 & 9.8 & 0.21 & 0.12 & 0.13 \\
\hline Probability & 0.01 & 0.01 & 0.01 & 0.05 & 0.05 & 0.05 \\
\hline \multicolumn{7}{|c|}{ Effect of Bio-Tonic levels } \\
\hline B1 (control) & $1569^{\mathrm{a}}$ & $2528^{\mathrm{a}}$ & $4097^{\mathrm{a}}$ & 2.59 & 2.36 & 2.44 \\
\hline$(0.5 \mathrm{Kg} /$ ton $) \mathrm{B} 2$ & $1472^{\mathrm{a}}$ & $2421^{b}$ & $3893^{b}$ & 2.39 & 2.28 & 2.32 \\
\hline B3 (1.0 Kg/ton) & $1487^{\mathrm{a}}$ & $2517^{\mathrm{a}}$ & $4004^{\mathrm{a}}$ & 2.31 & 2.21 & 2.24 \\
\hline B4 (1.5 Kg/ton) & $1416^{\mathrm{b}}$ & $2424^{\mathrm{b}}$ & $3840^{\mathrm{b}}$ & 2.08 & 2.05 & 2.06 \\
\hline SEM & 9.5 & 13.0 & 12.1 & 0.30 & 0.17 & 0.12 \\
\hline Probability & 0.05 & 0.05 & 0.05 & N.S & N.S & N.S \\
\hline \multicolumn{7}{|c|}{ Interaction effect between light regimes and Bio-Tonic levels } \\
\hline LI x B1 & $1800^{\mathrm{e}}$ & $2972^{\mathrm{f}}$ & $4772^{\mathrm{e}}$ & $3.0^{\mathrm{cd}}$ & $2.63^{\mathrm{d}}$ & 2.76 \\
\hline L1 x B2 & $1720^{\mathrm{de}}$ & $2964^{\mathrm{f}}$ & $4684^{\mathrm{e}}$ & $2.9^{\mathrm{c}}$ & $2.60^{\mathrm{d}}$ & 2.70 \\
\hline L1 x B3 & $1663^{d}$ & $3036^{\mathrm{f}}$ & $4699^{\mathrm{e}}$ & $2.7^{\mathrm{c}}$ & $2.56^{\mathrm{d}}$ & 2.61 \\
\hline L1 x B4 & $1747^{\mathrm{e}}$ & $2738^{\mathrm{e}}$ & $4485^{\mathrm{d}}$ & $2.6^{\mathrm{c}}$ & $2.34^{\mathrm{cd}}$ & 2.43 \\
\hline $\mathrm{L} 2 \times \mathrm{B} 1$ & $1415^{\mathrm{c}}$ & $2286^{\mathrm{c}}$ & $3701^{\mathrm{c}}$ & $2.5^{\mathrm{c}}$ & $2.23^{\mathrm{c}}$ & 2.33 \\
\hline $\mathrm{L} 2 \times \mathrm{B} 2$ & $1380^{\mathrm{bc}}$ & $2220^{\mathrm{b}}$ & $3600^{\mathrm{b}}$ & $2.3^{\mathrm{b}}$ & $2.20^{\mathrm{c}}$ & 2.24 \\
\hline $\mathrm{L} 2 \times \mathrm{B} 3$ & $1451^{\mathrm{c}}$ & $2169^{b}$ & $3621^{\mathrm{b}}$ & $2.3^{\mathrm{b}}$ & $2.10^{\mathrm{bc}}$ & 2.17 \\
\hline $\mathrm{L} 2 \times \mathrm{B} 4$ & $1401^{\mathrm{c}}$ & $2142^{\mathrm{ab}}$ & $3543^{b}$ & $2.1^{\mathrm{b}}$ & $2.10^{\mathrm{bc}}$ & 2.10 \\
\hline L3 x B1 & $1493^{c}$ & $2328^{\mathrm{c}}$ & $3820^{\mathrm{c}}$ & $2.3^{\mathrm{b}}$ & $2.20^{\mathrm{c}}$ & 2.24 \\
\hline $\mathrm{L} 3 \times \mathrm{B} 2$ & $1316^{\mathrm{b}}$ & $2080^{\mathrm{a}}$ & $3396^{\mathrm{a}}$ & $2.0^{\mathrm{b}}$ & $2.00^{\mathrm{b}}$ & 2.00 \\
\hline L3 x B3 & $1347^{b}$ & $2346^{\mathrm{c}}$ & $3693^{\mathrm{bc}}$ & $1.96^{\mathrm{b}}$ & $1.96^{\mathrm{b}}$ & 1.96 \\
\hline $\mathrm{L} 3 \times \mathrm{B} 4$ & $1101^{\mathrm{a}}$ & $2394^{\text {cd }}$ & $3495^{\mathrm{b}}$ & $1.58^{\mathrm{a}}$ & $1.76^{\mathrm{a}}$ & 1.70 \\
\hline SEM & 20.0 & 19.0 & 15.0 & 0.18 & 0.15 & 0.20 \\
\hline Probability & 0.01 & 0.05 & 0.01 & 0.01 & 0.05 & N.S \\
\hline
\end{tabular}

N.S. Not significant

a,b,c,d,e, f: means within the same column not having similar superscripts are significantly different $(\mathrm{P}<0.05)$ 
The birds fed on B1 diet had feed consumption higher than those fed on other Bio-Tonic levels in diet during all periods studied, the differences in this respect, were significant $(\mathrm{P}<0.05$, Table 3$)$.

Chicks fed on B1 level gave insignificantly higher feed conversion ratio than those other Bio-Tonic levels in diet (Table 3). These results suggest that birds fed on B4 were more efficient than those reared under other Bio-Tonic levels in diet. On the other hand, Abdel-Malak et al. (1995), El-Hindawy et al. (1996), El-Gendi et al. (2000) and Abdel-Malak et al. (1995) showed that feed consumption increased as the percentage of supplemented Bio-Tonic increased. It was evident that, adding BioTonic to broiler rations may improve the ration palatability and increase the amount of feed consumed.

There were significant differences $(\mathrm{P}<0.01)$ due to interaction effect between light regimes and Bio-Tonic level in diet on feed consumption during 1-4 and 1-7 weeks and feed conversion ratio during $1-4$ weeks period, significant $(\mathrm{P}<0.05)$ for feed consumption and feed conversion ratio during 4-7 weeks period and not significant for feed conversion ratio during 1-7 weeks period (Table 3). The treatment of L3 x B4 was the best in feed conversion at all periods studied.

\section{Carcass parameters}

Data tabulated in Table (4) illustrated that the broiler chicks reared under intermittent light regime gave percentage of dressing, liver, gizzard, heart and giblets non-significantly higher than either continuous or constant lighting program. While, abdominal fat was insignificantly higher for continuous than that constant and intermittent lighting program (Table 4). Similar results were reported by Al-Homidan (1994) and Al-Homidan and Petchey (2001), Stanley et al. (1997) and El-Neney (2003) who showed that the differences among light regimes (continuous, constant and intermittent light) on carcass weight, abdominal fat, heart weight, liver weight and dressing percentage were not significant. On the other hand, Kalamah (2002) reported that the absolute weight of heart was significantly lower in the chicks reared under constant compared with those exposed to either continuous or intermittent light, the weight of liver and spleen for birds grown under constant was significantly greater compared to the other light treatments, the photoperiods significantly affected the relative weight of liver and spleen, it did not significantly affect the relative weight of heart.

Table (4) show that chicks fed on B4 in diet gave insignificantly percentages of dressing, liver, gizzard, heart and giblets greater than that fed on the other Bio-Tonic treatments. An opposite trend was true for abdominal fat \% with B1 treatment (Table 4). In this respect, Ali (1999), Tawfeek and Marai (1997) and El-Hindawy et al. (1996) showed that the chicks fed diet supplemented with Yea-Sacc plus Bio-Tonic for long period (first 6 weeks of age) insignificantly improved most traits of carcass when compared to control diet.

On the other hand, El-Gendi et al. (2000) found that broiler chicks fed on dietary supplementation of Bio-Tonic and Zinc bacitracin showed significant increase in the total edible meat when compared with other level of different treatments applied. However, Abdel-Malak et al.(1995) reported that there were no significant differences among groups fed on different levels of Bio-Tonic on total edible protein of carcass, while giblets weight were significantly $(\mathrm{P}<0.05)$ different. 
Table 4. Effect of light regimes and bio-tonic (B) level on carcass parameters

\begin{tabular}{|c|c|c|c|c|c|c|}
\hline \multirow[t]{2}{*}{ Classification } & \multicolumn{6}{|c|}{ Carcass parameters } \\
\hline & $\begin{array}{c}\text { Dressing } \\
\%\end{array}$ & $\begin{array}{l}\text { Abdominal } \\
\text { Fat } \%\end{array}$ & $\begin{array}{c}\text { Liver } \\
\%\end{array}$ & $\begin{array}{c}\text { Gizzard } \\
\%\end{array}$ & $\begin{array}{l}\text { Heart } \\
\%\end{array}$ & $\begin{array}{c}\text { Giblets } \\
\%\end{array}$ \\
\hline \multicolumn{7}{|c|}{ Effect of light regimes } \\
\hline (L1) Continuous & 73.6 & 2.6 & 2.20 & 2.12 & 0.48 & 5.77 \\
\hline (L2) Constant & 69.7 & 2.2 & 2.14 & 2.16 & 0.49 & 5.70 \\
\hline (L3) Intermittent & 75.5 & 2.4 & 2.23 & 2.89 & 0.50 & 6.76 \\
\hline SEM & 2.0 & 0.30 & 0.06 & 0.11 & 0.03 & 0.12 \\
\hline Probability & N.S & N.S & N.S & N.S & N.S & N.S \\
\hline \multicolumn{7}{|c|}{ Effect of Bio-Tonic levels } \\
\hline B1 (control) & 71.7 & 2.3 & 2.21 & 2.10 & 0.47 & 5.79 \\
\hline$(0.5 \mathrm{Kg} /$ ton $) \mathrm{B} 2$ & 71.6 & 2.3 & 2.18 & 2.10 & 0.48 & 5.86 \\
\hline $\mathrm{B} 3(1.0 \mathrm{Kg} /$ ton $)$ & 73.4 & 2.1 & 2.18 & 2.18 & 0.49 & 6.20 \\
\hline B4 (1.5 Kg/ton) & 75.0 & 2.1 & 2.21 & 2.23 & 0.51 & 6.44 \\
\hline SEM & 1.12 & 0.25 & 0.12 & 0.12 & 0.02 & 0.40 \\
\hline Probability & N.S & N.S & N.S & N.S & N.S & N.S \\
\hline \multicolumn{7}{|c|}{ Interaction effect between light regimes and Bio-Tonic levels } \\
\hline LI x B1 & $73.5 b$ & $2.6 \mathrm{c}$ & 2.25 & 2.07 & 0.46 & 5.45 \\
\hline L1 x B2 & $72.7 b$ & $2.7 \mathrm{c}$ & 2.18 & 2.10 & 0.47 & 5.50 \\
\hline $\mathrm{L} 1 \times \mathrm{B} 3$ & $73.6 b$ & $2.5 \mathrm{bc}$ & 2.20 & 2.13 & 0.49 & 6.01 \\
\hline L1 x B4 & $74.5 \mathrm{c}$ & $2.6 \mathrm{c}$ & 2.22 & 2.17 & 0.49 & 6.10 \\
\hline L2 x B1 & $68.9 \mathrm{a}$ & $2.4 \mathrm{~b}$ & 2.19 & 2.10 & 0.47 & 5.35 \\
\hline $\mathrm{L} 2 \times \mathrm{B} 2$ & $69.5 \mathrm{a}$ & $2.3 b$ & 2.17 & 2.13 & 0.48 & 5.33 \\
\hline L2 x B3 & $70.0 \mathrm{a}$ & $2.1 \mathrm{~b}$ & 2.15 & 2.20 & 0.49 & 5.79 \\
\hline $\mathrm{L} 2 \times \mathrm{B} 4$ & $70.2 \mathrm{ab}$ & $2.0 \mathrm{~b}$ & 2.07 & 2.23 & 0.50 & 6.30 \\
\hline $\mathrm{L} 3 \times \mathrm{B} 1$ & $72.7 b$ & $2.0 \mathrm{~b}$ & 2.20 & 2.10 & 0.48 & 6.56 \\
\hline L3 x B2 & $72.5 b$ & $1.9 \mathrm{ab}$ & 2.18 & 2.06 & 0.48 & 6.75 \\
\hline $\mathrm{L} 3 \times \mathrm{B} 3$ & $76.7 \mathrm{c}$ & $1.6 \mathrm{a}$ & 2.20 & 2.22 & 0.49 & 6.80 \\
\hline $\mathrm{L} 3 \times \mathrm{B} 4$ & $80.2 \mathrm{~d}$ & $1.6 \mathrm{a}$ & 2.33 & 2.28 & 0.53 & 6.93 \\
\hline SEM & 1.5 & 0.33 & 0.09 & 0.13 & 0.06 & 0.10 \\
\hline Probability & 0.01 & 0.05 & N.S & N.S & N.S & N.S \\
\hline
\end{tabular}

N.S. Not significant

a,b,c and d means within the same column not having similar superscripts are significantly different $(\mathrm{P}<0.05)$

The interaction between photoperiod and Bio-Tonic level in diet in carcass parameters was highly significant $(\mathrm{P}<0.01)$ for dressing \%, significant $(\mathrm{P}<0.05)$ for percentages of abdominal fat, and not significant for percentages of liver, gizzard, heart and giblets (Table 4). Moreover, treatment L3 x B4 had the highest value of traits dressing, liver, gizzard, heart and giblets $\%$ and the lowest value in trait of abdominal fat $\%$

\section{Chemical composition of meat}

The meat moisture \% and crude protein (C.P) \% were insignificantly higher for birds exposed to intermittent than that exposed to either continuous or constant lighting programs. Moreover, birds exposed to constant light program had ether extract (E.E) \% significantly $(\mathrm{P}<0.05)$ greater than that exposed to intermittent and 
insignificant for continuous lighting program. Furthermore, crude ash (C.A) \% was nearly similar in all lighting programs used (Table 5).

Table 5. Effect of light regimes and bio-tonic (B) level on chemical composition of meat

\begin{tabular}{|c|c|c|c|c|}
\hline \multirow[t]{3}{*}{ Classification } & \multicolumn{4}{|c|}{ chemical composition of meat } \\
\hline & Moisture \% & C.P \% & E.E \% & C.A \% \\
\hline & \multicolumn{4}{|c|}{ Effect of light regimes } \\
\hline (L1) Continuous & 74.12 & 74.71 & $17.10 \mathrm{ab}$ & 5.12 \\
\hline (L2) Constant & 73.68 & 73.48 & $18.00 \mathrm{a}$ & 5.07 \\
\hline (L3) Intermittent & 74.63 & 75.35 & $16.10 \mathrm{~b}$ & 5.11 \\
\hline SEM & 0.27 & 0.30 & 0.27 & 0.05 \\
\hline Probability & N.S & N.S & 0.05 & N.S \\
\hline \multicolumn{5}{|c|}{ Effect of Bio-Tonic levels } \\
\hline B1 (control) & 7400 & 73.90 & 17.45 & 5.10 \\
\hline$(0.5 \mathrm{Kg} /$ ton $) \mathrm{B} 2$ & 74.06 & 74.03 & 17.24 & 5.11 \\
\hline B3 (1.0 Kg/ton) & 74.28 & 75.00 & 16.80 & 5.09 \\
\hline B4 (1.5 Kg/ton) & 74.22 & 75.12 & 16.68 & 5.08 \\
\hline SEM & 0.34 & 0.40 & 0.36 & 0.12 \\
\hline Probability & N.S & N.S & N.S & N.S \\
\hline \multicolumn{5}{|c|}{ Interaction effect between light regimes and Bio-Tonic levels } \\
\hline LI x B1 & 74.16 & 74.25 & $17.11 \mathrm{~b}$ & 5.12 \\
\hline L1 x B2 & 74.33 & 74.24 & $17.03 \mathrm{~b}$ & 5.13 \\
\hline L1 x B3 & 74.12 & 75.11 & $17.10 \mathrm{~b}$ & 5.06 \\
\hline L1 x B4 & 73.86 & 75.22 & $17.01 \mathrm{~b}$ & 5.11 \\
\hline $\mathrm{L} 2 \times \mathrm{B} 1$ & 73.82 & 73.32 & $18.52 d$ & 5.07 \\
\hline $\mathrm{L} 2 \times \mathrm{B} 2$ & 73.46 & 73.46 & $18.13 d$ & 5.04 \\
\hline L2 x B3 & 73.63 & 73.50 & $17.67 \mathrm{c}$ & 5.08 \\
\hline $\mathrm{L} 2 \times \mathrm{B} 4$ & 73.80 & 73.64 & $17.52 \mathrm{c}$ & 5.08 \\
\hline L3 x B1 & 74.00 & 74.12 & $16.72 b$ & 5.12 \\
\hline L3 $\times$ B2 & 74.40 & 74.39 & $16.55 \mathrm{ab}$ & 5.14 \\
\hline L3 x B3 & 75.10 & 76.26 & $15.62 \mathrm{a}$ & 5.12 \\
\hline L3 $x$ B4 & 75.00 & 76.64 & $15.50 \mathrm{a}$ & 5.04 \\
\hline SEM & 0.46 & 0.48 & 0.47 & 0.16 \\
\hline Probability & N.S & N.S & 0.05 & N.S \\
\hline
\end{tabular}

N.S. Not significant

a,b,c and $\mathrm{d}$ means within the same column not having similar superscripts are significantly different $(\mathrm{P}<0.05)$
C.P \% : Crude protein
E.E \% : Ether extract
C.A \% : Crude ash

Data presented in Table (5) illustrate that the differences among Bio-Tonic levels in all meat composition were not significant, and the averages were nearly similar (Table 5).

There were no significant differences due to interaction effect between light regimes and Bio-Tonic level in diet in all chemical compositions of meat studied except ether extract (E.E) \%, it was significant $(\mathrm{P}<0.05$, Table 5). Moreover, the lowest and highest value of ether extract $\%$ and crude protein $\%$ respectively was in the treatment of L3 x B4. 
Liver contents (lipids, protein and glycogen)

Data in Table (6) indicate that broiler chicks reared under intermittent light regime gave significantly $(\mathrm{P}<0.01$ and $\mathrm{P}<0.05)$ higher liver protein $\%$ and glycogen $(\mathrm{mg} / \mathrm{g})$ greater than that reared under both continuous and constant lighting programs. Moreover, chicks reared under continuous light regime had insignificantly liver lipids $\%$ higher than other photoperiods. This finding agrees with the results of El-Neney (2003) who demonstrated that at $7 \mathrm{wk}$ of age, birds subjected to continuous or intermittent light systems, exhibited more liver lipids (24.0 and $23.3 \%)$ than those under constant system (21.9\%), the effect of different light systems on protein level was highly significant at $7 \mathrm{wk}$ of age. In case of glycogen content, light systems (continuous, constant and intermittent light) did not affect liver glycogen content. These results were in disagreement with those obtained by Al-Homidan (1994).

Table 6. Effect of light regimes and bio-tonic (B) level on liver lipids, proteins and glycogen

\begin{tabular}{|c|c|c|c|}
\hline \multirow[t]{2}{*}{ Classification } & \multicolumn{3}{|c|}{ Liver } \\
\hline & Lipids\% & Proteins\% & Glycogen Mg/g \\
\hline \multicolumn{4}{|c|}{ Effect of light regimes } \\
\hline (L1) Continuous & 22.92 & $68.85 \mathrm{ab}$ & $0.96 \mathrm{~b}$ \\
\hline (L2) Constant & 23.42 & $66.85 \mathrm{~b}$ & $0.97 \mathrm{~b}$ \\
\hline (L3) Intermittent & 21.54 & $70.11 \mathrm{a}$ & $1.32 \mathrm{a}$ \\
\hline SEM & 0.45 & 0.86 & 0.18 \\
\hline Probability & N.S & 0.01 & 0.05 \\
\hline \multicolumn{4}{|c|}{ Effect of Bio-Tonic levels } \\
\hline B1 (control) & 23.02 & 67.22 & $0.98 \mathrm{~b}$ \\
\hline$(0.5 \mathrm{Kg} /$ ton $) \mathrm{B} 2$ & 23.12 & 68.27 & $0.97 \mathrm{~b}$ \\
\hline B3 $(1.0 \mathrm{Kg} /$ ton $)$ & 22.20 & 69.22 & $1.14 \mathrm{a}$ \\
\hline B4 (1.5 Kg/ton) & 22.14 & 69.71 & $1.24 \mathrm{a}$ \\
\hline SEM & 0.52 & 0.66 & 0.15 \\
\hline Probability & N.S & N.S & 0.05 \\
\hline \multicolumn{4}{|c|}{ Interaction effect between light regimes and Bio-Tonic levels } \\
\hline LI x B1 & 22.98 & $68.98 \mathrm{~b}$ & 0.97 \\
\hline $\mathrm{L} 1 \times \mathrm{B} 2$ & 23.00 & $69.00 \mathrm{~b}$ & 0.96 \\
\hline L1 x B3 & 22.75 & $68.66 \mathrm{~b}$ & 0.94 \\
\hline $\mathrm{L} 1 \times \mathrm{B} 4$ & 22.98 & $68.76 \mathrm{~b}$ & 0.98 \\
\hline $\mathrm{L} 2 \times \mathrm{B} 1$ & 24.00 & $65.75 \mathrm{a}$ & 0.99 \\
\hline $\mathrm{L} 2 \times \mathrm{B} 2$ & 23.90 & $66.77 \mathrm{ab}$ & 0.098 \\
\hline L2 x B3 & 23.00 & $67.00 \mathrm{~b}$ & 0.95 \\
\hline $\mathrm{L} 2 \times \mathrm{B} 4$ & 22.79 & $67.88 \mathrm{~b}$ & 0.97 \\
\hline L3 x B1 & 2209 & $66.93 b$ & 0.98 \\
\hline $\mathrm{L} 3 \times \mathrm{B} 2$ & 22.60 & $69.03 b$ & 0.96 \\
\hline $\mathrm{L} 3 \times \mathrm{B} 3$ & 20.82 & $72.00 \mathrm{c}$ & 1.53 \\
\hline L3 x B4 & 20.65 & $72.50 \mathrm{c}$ & 1.76 \\
\hline SEM & 0.53 & 0.93 & 0.22 \\
\hline Probability & N.S & 0.01 & N.S \\
\hline
\end{tabular}

N.S. Not significant

$\mathrm{a}, \mathrm{b}$ and $\mathrm{c}$ means within the same column not having similar superscripts are significantly different $(\mathrm{P}<0.05)$ 
Table (6) shows that chicks fed on B4 level had insignificantly lower and higher liver lipids and protein \% than other levels, respectively. B4 and B3 had significantly $(\mathrm{P}<0.05)$ glycogen $(\mathrm{mg} / \mathrm{g})$ greater than the other Bio-Tonic levels.

The interaction between light regimes and Bio-Tonic level in diet on liver protein $\%$ was highly significant $(\mathrm{P}<0.01)$, the highest value was in treatments L3 x B4. There were no significant differences due to interaction between light regimes and Bio-Tonic level in diet on liver lipids $\%$ and liver glycogen $(\mathrm{mg} / \mathrm{g})$ Table (6). Moreover, the lowest and highest value in liver lipids \% and liver glycogen $(\mathrm{mg} / \mathrm{g})$ respectively was in treatment L3 x B4.

\section{Blood plasma constituents}

Chicks exposed to intermittent lighting program had insignificantly higher plasma total protein, albumin, alkaline phosphatase, and GOT than that exposed to either continuous or constant lighting programs.

Plasma total lipids were insignificantly higher for constant than either continuous or intermittent lighting program, while, cholesterol had the lowest (insignificant) value in intermittent lighting program compared with other light regimes. Plasma GPT was insignificantly higher in chicks exposed to continuous than that exposed to either constant or intermittent lighting programs.

Plasma hormones of T3 and G.H were, in general, greater in chicks exposed to intermittent lighting program than that exposed to either continuous or constant lighting programs, the differences, in this respect, were significant $(\mathrm{P}<0.05)$ for $\mathrm{T} 3$, and not significant for GH. However, plasma T4 hormone was nearly similar in all photoperiod programs (Table 7). These results are in agreement with those reported by El-Neney (2003) who showed that there was no significant differences due to light regimes on plasma total protein, the broiler chicks reared under intermittent lighting system (4L: 8D : 4L : 8D) had insignificantly total protein and significant $(\mathrm{P}<0.05)$ plasma albumin higher than that reared under continuous lighting system (23L: 1D). Birds with constant and intermittent lights had higher GH than that of continuous light. Also, Buyse et al., (1998) reported that birds under intermittent light have higher plasma T3 concentrations than those exposed to constant light. On the other hand, Buyse et al. (1997), Buys et al. (1998) and El-Neney (2003) who found that broiler chicks reared under intermittent lighting system (4L: 8D : 4L : 8D) had plasma cholesterol higher than that reared under continuous lighting system (23L: $1 D)$, the differences in this respect were highly significant $(\mathrm{P}<0.01)$.

Light systems, especially intermittent light, showed the most highly concentration of $\left(\mathrm{T}_{3}\right)$ among the three light treatments. This may be due to that, the reduction of light may be associated with metabolic changes during darkness. Birds with intermittent lights had higher GH than those of constant and continuous light. These results are in agreement with those reported by (Buyse et al., 1997 and Buys et al., 1998) that Plasma growth hormone levels in birds reared under intermittent light were higher than those of constant light. Intermittent light manifesting compensatory growth have higher mean plasma growth hormone levels than their age-matched counterparts (Kuhn et al., 1996).

The chicks fed on B4 level had plasma total protein and albumin had significantly $(\mathrm{P}<0.05)$ higher than those fed on the other levels of Bio-Tonic., for plasma albumin, and not significant for plasma total protein. 
Chicks fed B4 had insignificantly higher plasma total lipids, and significantly $(\mathrm{P}<0.05)$ lower cholesterol than those fed on different levels of Bio-Tonic in diet.

Plasma alkaline phosphatase activity and GOT were, in general, insignificantly higher in chicks fed on B4 level in diet than the other levels of Bio-Tonic in diet. An opposite trend was true for GPT with B1 (Table 7).

Table 7. Effect of light regimes and bio-tonic (B) level on blood plasma constituents

\begin{tabular}{|c|c|c|c|c|c|}
\hline \multirow[t]{2}{*}{ Classification } & \multicolumn{5}{|l|}{ Plasma } \\
\hline & $\begin{array}{l}\text { Total } \\
\text { protein } \\
\text { g } / 100 \mathrm{ml} \\
\end{array}$ & $\begin{array}{l}\text { Albumin } \\
\text { g / 100ml }\end{array}$ & $\begin{array}{l}\text { Total } \\
\text { Lipids } \\
\text { g/L }\end{array}$ & $\begin{array}{l}\text { Cholesterol } \\
\mathrm{Mg} / \mathbf{1 0 0 m l}\end{array}$ & $\begin{array}{l}\text { Alkalin } \\
\text { phosphatase } \\
\text { U/L }\end{array}$ \\
\hline \multicolumn{6}{|c|}{ Effect of light regimes } \\
\hline (L1) & 4.62 & 1.76 & 16.63 & 111 & 222 \\
\hline \multicolumn{6}{|l|}{ Continuous } \\
\hline (L2) Constant & 4.00 & 1.81 & 17.00 & 110 & 225 \\
\hline (L3) & 4.77 & 1.94 & 15.00 & 107 & 230 \\
\hline \multicolumn{6}{|l|}{ Intermittent } \\
\hline SEM & 0.38 & 0.19 & 0.48 & 0.61 & 0.44 \\
\hline Probability & N.S & N.S & N.S & N.S & N.S \\
\hline \multicolumn{6}{|c|}{ Effect of Bio-Tonic levels } \\
\hline B1 (control) & 4.22 & $1.65 \mathrm{~b}$ & 16.83 & $113 \mathrm{a}$ & 204 \\
\hline$(0.5 \mathrm{Kg} /$ ton $) \mathrm{B} 2$ & 4.38 & $1.75 \mathrm{~b}$ & 16.36 & $109 \mathrm{a}$ & 217 \\
\hline B3 $(1.0 \mathrm{Kg} /$ ton $)$ & 4.58 & $1.92 \mathrm{a}$ & 16.00 & $107 \mathrm{~b}$ & 235 \\
\hline $\mathrm{B} 4(1.5 \mathrm{Kg} /$ ton $)$ & 4.64 & $2.03 \mathrm{a}$ & 15.56 & $106 \mathrm{~b}$ & 247 \\
\hline SEM & 0.40 & 0.20 & 0.50 & 1.20 & 0.56 \\
\hline Probability & N.S & 0.05 & N.S & 0.05 & N.S \\
\hline \multicolumn{6}{|c|}{ Interaction effect between light regimes and Bio-Tonic levels } \\
\hline LI x B1 & $4.45 \mathrm{bc}$ & $1.56 \mathrm{a}$ & 16.85 & $117 \mathrm{c}$ & 205 \\
\hline $\mathrm{L} 1 \times \mathrm{B} 2$ & $4.63 \mathrm{c}$ & $1.62 \mathrm{a}$ & 16.75 & $110 \mathrm{~b}$ & 213 \\
\hline L1 x B3 & $4.66 \mathrm{c}$ & $1.86 \mathrm{~b}$ & 16.56 & $109 b$ & 232 \\
\hline L1 x B4 & $4.72 \mathrm{c}$ & $2.00 \mathrm{c}$ & 16.36 & $107 \mathrm{a}$ & 238 \\
\hline $\mathrm{L} 2 \times \mathrm{B} 1$ & $3.99 \mathrm{a}$ & $1.65 \mathrm{a}$ & 17.22 & $113 b$ & 200 \\
\hline $\mathrm{L} 2 \times \mathrm{B} 2$ & $3.97 \mathrm{a}$ & $1.70 \mathrm{ab}$ & 17.19 & $111 b$ & 219 \\
\hline L2 x B3 & $3.98 \mathrm{a}$ & $1.89 \mathrm{~b}$ & 17.20 & $108 \mathrm{ab}$ & 235 \\
\hline $\mathrm{L} 2 \times \mathrm{B} 4$ & $4.00 \mathrm{a}$ & $1.99 \mathrm{bc}$ & 16.30 & $109 b$ & 247 \\
\hline L3 x B1 & $4.23 b$ & $1.75 \mathrm{~b}$ & 16.42 & $110 \mathrm{~b}$ & 207 \\
\hline L3 x B2 & $4.54 c$ & $1.92 \mathrm{~b}$ & 15.13 & $108 \mathrm{ab}$ & 220 \\
\hline L3 $x$ B3 & $5.11 \mathrm{~d}$ & $2.00 \mathrm{c}$ & 14.20 & $105 \mathrm{a}$ & 237 \\
\hline L3 $\times$ B4 & $5.21 \mathrm{~d}$ & $2.10 \mathrm{c}$ & 14.02 & $103 a$ & 255 \\
\hline SEM & 0.52 & 0.23 & 0.56 & 0.75 & 0.80 \\
\hline Probability & 0.05 & 0.05 & N.S & 0.01 & N.S \\
\hline
\end{tabular}

N.S. Not significant

$\mathrm{a}, \mathrm{b}, \mathrm{c}$ and $\mathrm{d}$ means within the same column not having similar superscripts are significantly different $(\mathrm{P}<0.05)$ 
Table 7. Cont.

\begin{tabular}{|c|c|c|c|c|c|}
\hline \multirow[t]{2}{*}{ Classification } & \multicolumn{5}{|c|}{ Plasma } \\
\hline & $\begin{array}{c}\text { GOT } \\
\text { U/I }\end{array}$ & $\begin{array}{c}\text { GPT } \\
\text { U/I }\end{array}$ & $\begin{array}{c}\text { T } 3 \\
\text { ng/dl }\end{array}$ & $\begin{array}{c}\text { T } 4 \\
\mathrm{ng} / \mathrm{dl}\end{array}$ & $\begin{array}{c}\text { G.H } \\
\text { Iu/ml }\end{array}$ \\
\hline \multicolumn{6}{|c|}{ Effect of light regimes } \\
\hline (L1) Continuous & 30 & 6.2 & $135 \mathrm{ab}$ & 1.79 & 0.299 \\
\hline (L2) Constant & 29 & 4.7 & $132 \mathrm{~b}$ & 1.75 & 0.324 \\
\hline (L3) Intermittent & 31 & 5.6 & $137 \mathrm{a}$ & 1.76 & 0.333 \\
\hline SEM & 0.05 & 0.04 & 0.21 & 0.30 & 0.05 \\
\hline Probability & N.S & N.S & 0.05 & N.S & N.S \\
\hline \multicolumn{6}{|c|}{ Effect of Bio-Tonic levels } \\
\hline B1 (control) & 26 & 6.1 & $136 \mathrm{~b}$ & 1.79 & $0.230 \mathrm{c}$ \\
\hline$(0.5 \mathrm{Kg} /$ ton $) \mathrm{B} 2$ & 30 & 5.9 & $134 \mathrm{c}$ & 1.79 & $0.356 \mathrm{~b}$ \\
\hline B3 (1.0 Kg/ton) & 31 & 5.7 & $137 \mathrm{~b}$ & 1.80 & $0.370 \mathrm{~b}$ \\
\hline B4 (1.5 Kg/ton) & 33 & 5.8 & $140 \mathrm{a}$ & 1.82 & $0.385 \mathrm{a}$ \\
\hline SEM & 0.07 & 0.03 & 0.18 & 0.61 & 0.10 \\
\hline Probability & N.S & N.S & 0.01 & N.S & 0.05 \\
\hline \multicolumn{6}{|c|}{ Interaction effect between light regimes and Bio-Tonic levels } \\
\hline LI x B1 & 27 & 7.0 & $134 \mathrm{a}$ & 1.80 & $0.320 \mathrm{~b}$ \\
\hline L1 x B2 & 29 & 5.9 & $134 \mathrm{a}$ & 1.79 & $0.327 \mathrm{bc}$ \\
\hline $\mathrm{L} 1 \times \mathrm{B} 3$ & 30 & 5.8 & $137 \mathrm{ab}$ & 1.83 & $0.347 \mathrm{c}$ \\
\hline L1 x B4 & 33 & 5.6 & $138 b$ & 1.85 & $0.367 \mathrm{~d}$ \\
\hline L2 x B1 & 25 & 7.1 & $132 \mathrm{a}$ & 1.79 & $0.298 \mathrm{a}$ \\
\hline $\mathrm{L} 2 \times \mathrm{B} 2$ & 28 & 6.2 & $133 a$ & 1.77 & $0.323 b$ \\
\hline L2 x B3 & 30 & 4.9 & $135 a$ & 1.80 & $0.339 \mathrm{c}$ \\
\hline L2 x B4 & 31 & 5.8 & $138 b$ & 1.83 & $0.354 \mathrm{~cd}$ \\
\hline L3 x B1 & 26 & 6.9 & $137 \mathrm{ab}$ & 1.81 & $0.345 \mathrm{c}$ \\
\hline $\mathrm{L} 3 \times \mathrm{B} 2$ & 32 & 5.7 & $141 \mathrm{~b}$ & 1.78 & $0.360 \mathrm{~d}$ \\
\hline L3 x B3 & 33 & 5.3 & $147 \mathrm{c}$ & 1.83 & $0.386 \mathrm{ed}$ \\
\hline L3 x B4 & 35 & 5.4 & $150 \mathrm{c}$ & 1.87 & $0.395 \mathrm{e}$ \\
\hline SEM & 0.08 & 0.04 & 0.23 & 0.75 & 0.15 \\
\hline Probability & N.S & N.S & 0.05 & N.S & 0.05 \\
\hline
\end{tabular}

N.S. Not significant

$\mathrm{a}, \mathrm{b}, \mathrm{c}$ and $\mathrm{d}$ means within the same column not having similar superscripts are significantly different $(\mathrm{P}<0.05)$

Plasma albumin and hormones of T3, T4 and GH were, in general, greater in chicks fed on B4 level in diet than the other levels of Bio-Tonic in diet (Table 7), the differences, in this respect, were highly significant $(\mathrm{P}<0.01)$ for plasma $\mathrm{T} 3$, significant $(\mathrm{P}<0.05)$ for Plasma $\mathrm{GH}$ and albumin and insignificant for plasma $\mathrm{T} 4$ (Table 7). In this respect, El-Gendi et al. (2000) and Abdel-Malak et al. (1995) who reported that the means values of total protein, albumin, globulin, alkaline phosphatase in blood serum increased, while, GOT and GPT in blood serum decreased for broiler chicks fed on Bio-Tonic. They added that serum blood of total lipids and cholesterol were not significantly affected by this feed additive (BioTonic). Moreover, Abdel-Azeem (2002) reported that biological feed additive for broiler chicks diet did not have a significant effect on liver function (GOT and GPT). 
While, chicks fed on diets supplemented with biological feed additives showed higher values of blood total protein, albumin (A) and globulin (G). But, lower values of $\mathrm{A} / \mathrm{G}$ ratio, cholesterol and total lipids were observed when compared with the control group.

On the other hand, Poo and Millan (1990) and Tawfeek et al. (1993) observed that serum total protein, albumin $(\mathrm{A})$, globulin $(\mathrm{G}), \mathrm{A} / \mathrm{G}$ ratio, total lipids and thyroid hormones were not significantly affected by biological feed additive for broiler chick's diet.

The effect of interaction between photoperiod and Bio-Tonic level on plasma cholesterol was highly significant $(\mathrm{P}<0.01)$ and the lowest value of plasma cholesterol was in treatment L3 x B4, significant $(\mathrm{P}<0.05)$ for plasma total protein, albumin and plasma hormones of T3 and G.H, and the highest value of the previous traits was in treatment L3 x B4 and not significant for plasma total lipids, alkaline phosphatase activity, GOT , GPT and T4 hormones with the lowest value in treatment L3 x B4 of plasma total lipids, GPT and T4, moreover, the highest value in treatment L3 x B4 of traits alkaline phosphatase activity and GOT.

We recommend using the treatment L3 x B4 in broiler diet because it enhance body weight, body weight gain, meat composition and carcass traits, also decrease cholesterol, total lipids and increase total protein in the liver and plasma. All the previous characteristics increase the economic profit in broiler farmers.

\section{REFERENCES}

Al-Homidan, A.A., 1994. Effect of light regimes and feed frequency on performance of two commercial broiler strains. M. Sc. Thesis, Dep. Anim. Prod. College of Agric. K.S.U., Riyadh, K.S.A.

Al-Homidan, A.A. and A.M. Petchey, 2001. The effects of length and color of light regimes on performance and carcass characteristics of broiler chickens. Egypt, Poult. Sci. Vol. 21 (II): 549-566.

AOAC., 1990. Association of Official Agricultural Chemists. Methods of Analysis. Washington D. C.

Abdel-Malak, N.Y., Abdel-Malak, M.S., El-Gendi, G.M. and Emily, F. Naguib, 1995. Effect of different levels of herbal feed additive on broiler performance in relation to some metabolic functions. Egypt. Poult. Sci. J., 15: 111-139.

Abdel-Azeem, F. 2002. Digestion, neomycin and yeast supplementation in broiler diets under Egyptian summer conditions. Egypt. Poult. Sci. 22: 235-257.

Ali, M.A., 1999. Effect of probiotics addition to broiler rations on performance and some blood constituents. Egypt.Poult. Sci. J., 19 (1): 161-177.

AOAC., 1990. Association of Official Agricultural Chemists. Methods of Analysis. Washington D. C.

Bardley, G.L., T.F. Sava and K.J. Timm, 1994. The effects of supplementing diets with Saccharomyces cerevisiae Var boulardi on male poultry performance and ideal morphology. Poult. Sci. 73 (11): 1766-1770.

Buckland, R.B., A. Goldrosen and D.E. Bragg, 1971. Interaction of strain, density and ration with two light systems on broiler performance. Can. J. Anim. Sci. 51 : 613-619. 
Buys, N.,J. Buyse, M. Hassanadeh-Ladmakhi and E. Decuypere, 1998. Intermittent lighting reduces the incidence of ascites in broilers: An interaction with protein content of feed performance and the endocrine system. Poult. Sci.77:54-61.

Buyse, J., D. S. Adelsohn, E. Decuypere and C.G. Scanes, 1993. Diurnal-nocturnal changes in food intake, gut storage of ingesta, food transit time and metabolism in growing broiler chickens : a model for temporal control of energy balance. $\mathrm{Br}$. Poult. Sci. 34 : 699-709.

Buyse, J., E. Decuypere and J. D. Veldhuis, 1997. Compensatory growth of broiler chickens is associated with an enhanced pulsatile growth hormone $(\mathrm{GH})$ secretion: Preferential amplification of GH secretory burst mass. Br. Poult. Sci. 38: 291296.

Buyse, J., E.R. Kuhn and E. Decuypere, 1996. The use of intermittent lighting in broiler raising. 1. The effect on broiler performance, and on efficiency of dietary nitrogen retention. Poult. Sci. $75: 589-594$.

Classen, H.L., and C. Riddell, 1989. Photoperiodic effects on performance and leg abnormalities in broiler chickens. Poult. Sci. 68 : 873-879.

Classen, H. L., C. Riddell and F. E. Robinson, 1991. Effect of increasing photoperiod length on performance and health of broiler chickens. Br. Poult. Sci. 32: $21-29$.

Der Vies, J. V. 1954. Two methods for the determination of glycogen in liver. Biochem. J. 57: 410.

El-Gendi, G.M., F.A. Ismail and S.M. Aggoury, 1994. Effect of cocci-Nel and Lomoton dietary supplementation as herbal growth promoters on reproductive performance in broiler. Annual of Agric. Sci. Moshtohor, 32 (3): 1511-1528.

El-Gendi, G.M., A.F. Soliman and A.F. Habib, 2000. Evaluating four feed additives for improving productive and metabolic performance of broiler chicks. Egypt.Poult. Sci. J., 20 (1): 103-122.

El-Hindawy, M.M., M.I. Tawfeek and S.A. Osman, 1996. Effect of some biological feed additives for different periods on performance of broiler chicks. Zagazig J. of Agric. Res., 23 (5): 773-783.

El-Neney, B.A., 2003. Effect of light regimes and feed frequencies on broiler performance under Egyptian conditions. Ph.D. Thesis, Faculty of Agric., Minufiya Univ., Egypt.

Kalamah, M.A., 2002. Effect of different photoperiod regimens on growth performance and some physiological responses of different broiler strains. Minufiya J. Agric. Res. 27 (3): 695-715.

Kuhn, E. R., V.M. Darras, C. Gysemans, E. Decuypere, L. R. Berghman and J. Buyse, 1996. The use of intermittent lighting in broiler raising. 2- Effects on the somatotrophic and thryoid axes and on plasma testosterone levels. Poult. Sci. 75: 595- 600 .

Laster, C.P., F.J. Hoerr, S.F. Bilgili and S.A. Kincaid,. 1999. Effects of dietary roxarsone supplementation, lighting program, and season on the incidence of leg abnormalities in broiler chickens. Poult. Sci. 78: 197- 203.

Liu, G., E.A. Dunnington and P.B. Siegel, 1995. Correlated responses to long-term divergent selection for eight-week body weight in chickens: growth, sexual maturity and egg production. Poult. Sci. 74: 1259-1268.

Namur, A.P., J. Morel and H. Bichek, 1988. Compound animal feed and feed additives. In Deboer, F., H. BICHEL, eds. Livestock feed resources and feed evaluation in Europe. Elsevier. Sci. Publ., Amsterdam. 
Ohtani, S. and S. Leesont, 2000. The effect of intermittent lighting on metabolizable energy intake and heat production of male broilers. Poult. Sci. 79 : $167-171$.

Osman, S.M., 1996. Responses of broiler chicks for some dietary supplementation. M.Sc., Thesis, Fuculty of Agric., Zagazig Univ., Egypt.

Poo, M.E. and N. Millan, 1990. Effect of dietary concentration of yeast (Saccharomyces crlesbergensis) recovered from beer in male Warren chicks. Archivos-Latinoamericanos-de Nutrition. 40 (1): 95-106.

Renden, J.A., E.T. Jr. Moran and S.A. Kincaid, 1996. Lighting programs for broilers that reduce leg problems without loss of performance or yield. Poult. Sci. 75: $1345-1350$.

Savory, C.J., 1980. Diurnal feeding patterns in domestic fowl : a review. Applied Animal Ethology. 6 : 71-82.Smith, M.O. 1994. Effects of electrolyte and lighting regimen on growth of heat distressed broilers. Poult .Sci. $73: 350-353$.

SPSS., 1984. By SPSS INC., North Uichigan. U. S. A..

Stanley, V.G., J. Gutierrez, A.L. Parks, S.A. Rhoden, H. Chukwu, C. Gray and W.F. Krueger, 1997. Relationship between age of commercial broiler chickens and response to photostimulation. Poult .Sci. $76: 306-310$.

Tawfeek, M.I. and I.F. Marai, 1997. Use of probiotics in small animals production. International Conference on Animal, Poultry and Rabbit Production and Health. Institute of Efficient Productivity, Zagazig Univ., Zagazig, Egypt., 2-4 September.

Tawfeek, M.I., K.A.O. Yamani, A.A. Rashwan and Soad, S. Ahmed, 1993. Growth performance, carcass traits and blood constituents in broiler chicks as affected by genotype, dietary protein sources and feed additives. J. of Agric. Sci., Mansoura Univ., 18 (18): 2279-2289.

Weaver, K.W.D., Jr., W.L. Beane and J.A. Cherry, 1982. Effect of light, feed space, stocking density and dietary energy on broiler performance. Poult .Sci. $61: 33$ 37.

Weaver, K.W.D., Jr. and P.B. Siegel, 1968. Photoperiodism as a factor in feeding rhythms of broiler chickens. Poult .Sci. $47: 1148$. 
تحسين الصفات الإنتاجية وإلفسيولوجية لبدارى التسمين بإستخدام بعض نظم الاضاءة

والإضافات الغذائية

' الدمد محمود سليمان'، باتعة أحمد النتى'، مرضى عبد العظيم قلمة' ، علاء كمال علم الدين'

أجريت هذه الدراسة فى محطة أبحاث الدواجن- قسم أنتاج الدواجن - كلية الزراعة- جامعة المنوفية.

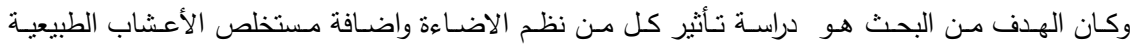

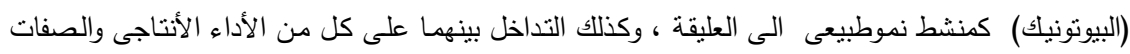

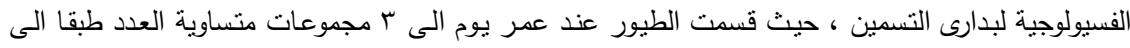

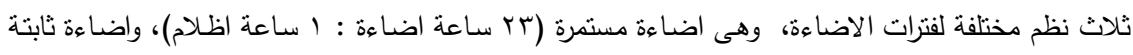

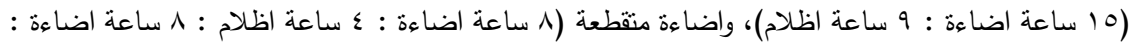

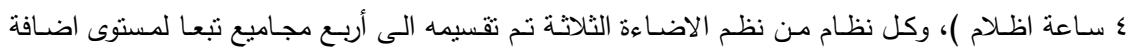

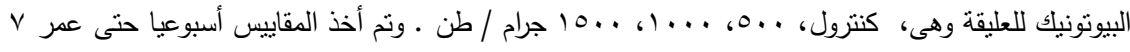

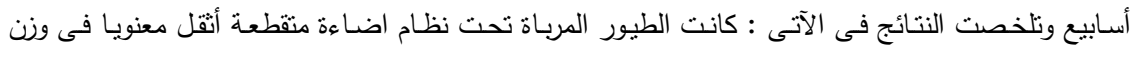

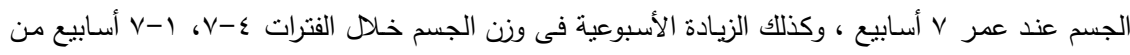

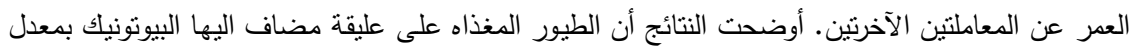

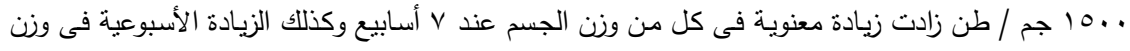

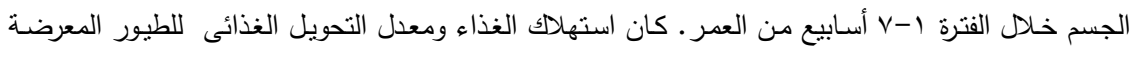

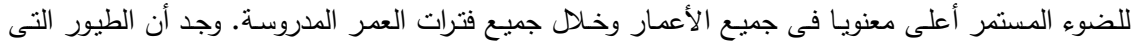

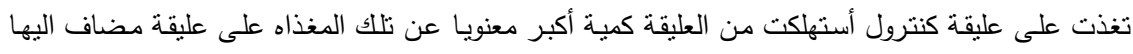

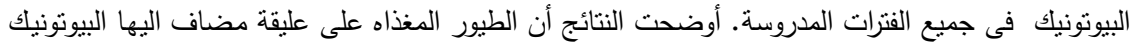

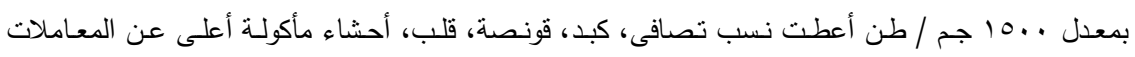

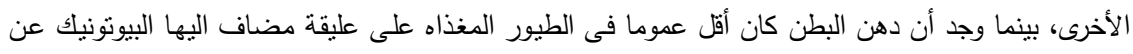

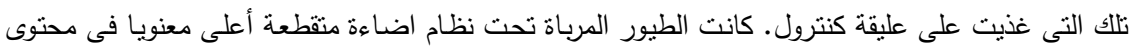

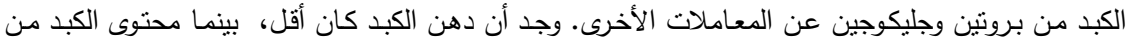

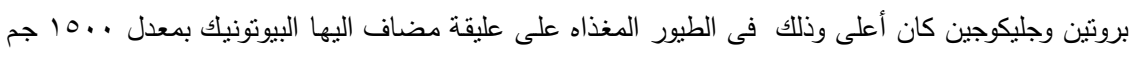

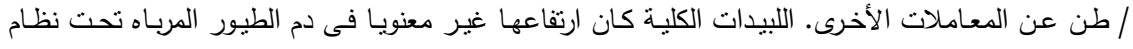

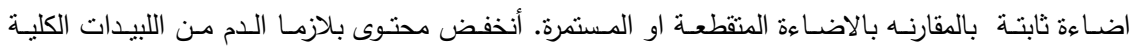

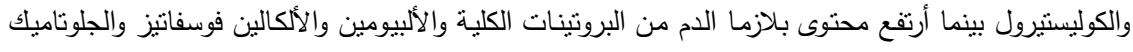


أوكسال أسيتك أسد الناقل لمجموعة الأمين (GOT) وهرمونات ) (T3 , T4, GH) فى دم الطيور المغذاة على البيوتونيك بمعدل .10 جم / طن عن المعدلات الأخرى. وجد أن هناك فروقا معنوية راجعة للتداخل بين

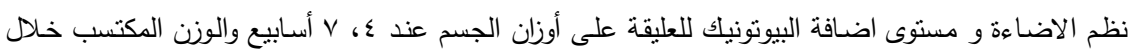

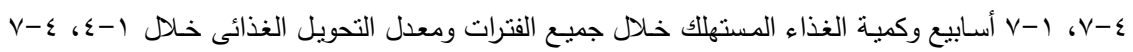
أسـابيع ومحتوى الكبد من البروتين ونسب التصافى ودهن البطن ومحتوى بلازما الدم من البروتينات الكلية والألبيومين والكوليستيرول وهرمونى (T3 , GH). بصفة عامـة ، يمكن أن نوصى باستخدام نظـام الاضـاءة المتقطعة مـع اضـافة مستخلص الأعشاب الطبيعيـة

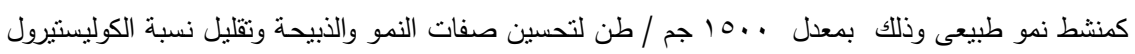

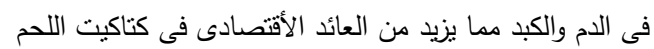

